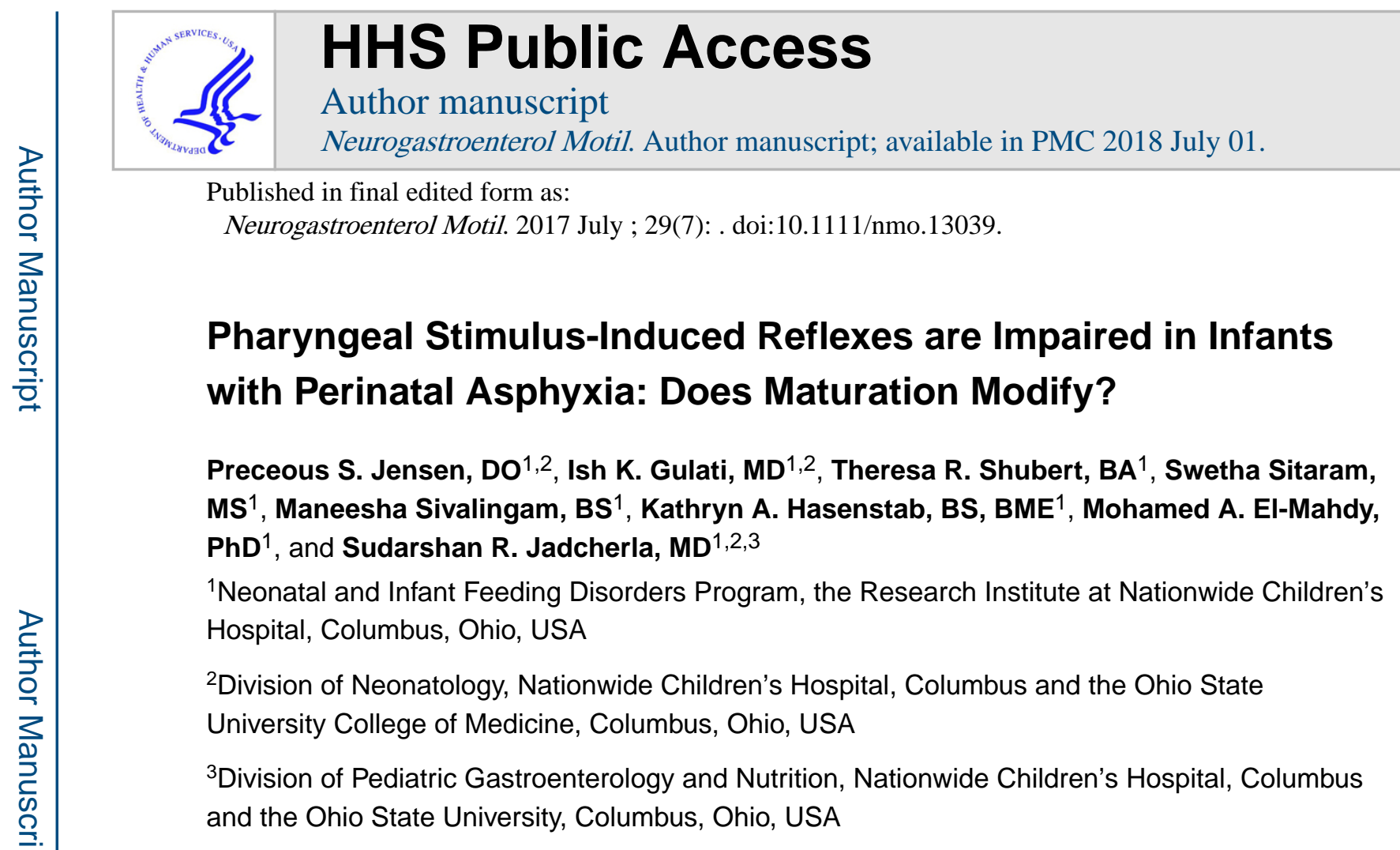

\title{
Abstract
}

Background-Development of pharyngo-esophageal protective reflexes among infants with hypoxic ischemic encephalopathy (HIE) is unclear. Our aim was to distinguish these reflexes from controls and examine the maturational changes in HIE infants.

\begin{abstract}
Methods-We evaluated 14 HIE infants (7 males) at 41.4 \pm 0.6 (HIE Time-1) and 46.5 \pm 0.6 (HIE Time-2) weeks postmenstrual age (PMA). 7 controls ( 3 males) were evaluated at $43.5 \pm 1.3$ weeks PMA. Graded pharyngeal stimulation with liquids $(0.1,0.3,0.5 \mathrm{~mL}$ in triplicate) concurrent with high resolution manometry were used to analyze sensory-motor components of pharyngeal reflexive swallowing (PRS), upper esophageal sphincter (UES) contractile reflex (PUCR), and esophageal body characteristics. Linear mixed and generalized estimating equation models were used for comparison among groups.
\end{abstract}

Key Results-Compared to controls, HIE infants (Time-1 and Time-2) exhibited decreased number of pharyngeal peaks and latency to terminal swallow. HIE Time-1 infants showed increased UES resting tone and distal latency, compared to controls and HIE Time-2. Contractile vigor was increasingly abnormal during maturation, compared to healthy controls. Threshold

\footnotetext{
Correspondence and Reprint Requests: Sudarshan R. Jadcherla, MD, FRCPI, DCH, AGAF, Division of Neonatology, Innovative Feeding Disorders Research Program, Center for Perinatal Research, Nationwide Children's Hospital Research Institute, 700 Children's Drive, Columbus, OH 43205, Phone: (614) 355-6643; Fax: (614) 355-6675, sudarshan.jadcherla@nationwidechildrens.org. Disclosure: No conflicts of interest to declare

Competing Interests: The authors have no competing interests AUTHOR CONTRIBUTIONS:

SRJ is the Principal Investigator and provided concept and design of paradigms, IRB process, and securing funding. PSJ, IKG, TRS, $\mathrm{KAH}, \mathrm{MS}$, and SRJ have all participated in performing manometry studies. PSJ, IKG, TRS, MS, KAH, and SRJ have provided manometry data analysis, validation, and interpretation. SS has provided statistical analysis, MAE has provided statistical validation. PSJ, TRS, SS, KAH, and MAE created figures and tables. PSJ and TRS have contributed to thorough literature review. All authors have contributed to manuscript writing, editing, and approval of final version.
} 
volumes and frequency distribution of primary responses (PRS: PUCR: None) were not significant among all groups.

Conclusions \& Inferences-Compared to controls, HIE infants display significant hypertonicity of skeletal muscle components, impairment of pharyngeal provocation induced reflexes and smooth muscle contractile vigor, reflecting poor propagation with maturation. These mechanisms may be responsible for inadequate clearance of secretions, ascending refluxate, and oropharyngeal bolus in HIE infants.

\section{Abbreviated abstract}

We evaluated pharyngo-esophageal protective reflexes following pharyngeal stimulation in hypoxic ischemic encephalopathy (HIE) infants studied longitudinally. High resolution manometry analysis showed that these reflexes are impaired in HIE infants which may be responsible for inadequate clearance of secretions, ascending refluxate, and oropharyngeal bolus. Strategies to optimize these reflexes are needed to ensure aerodigestive safety during oral feeding.

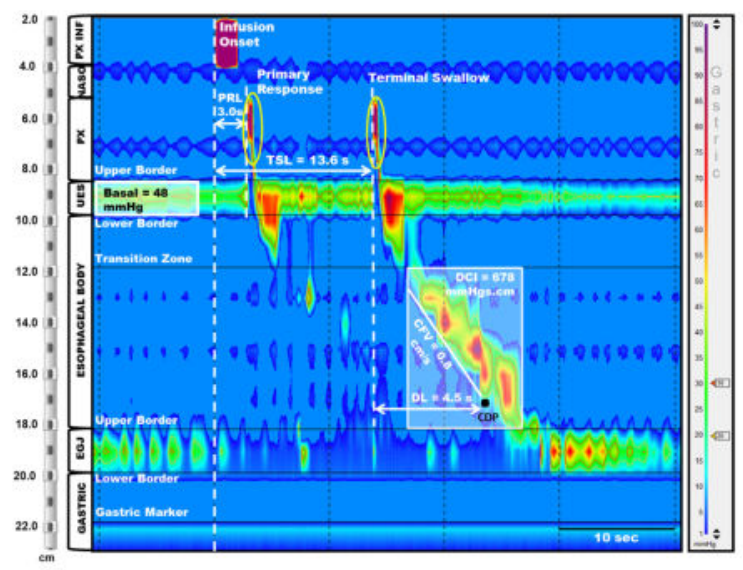

\section{Keywords}

premature infant; asphyxia; swallowing; esophageal motility

\section{Introduction}

The prevalence of perinatal asphyxia varies from 1-4 to $1-9$ per 1000 live births in developed countries and developing countries, respectively $(1,2)$. Perinatal asphyxia leads to decreased cerebral blood flow and oxygenation to the brain, causing hypoxic ischemic encephalopathy (HIE). Approximately, 65\% of perinatal asphyxia survivors suffer feeding impairment that may require a gastrostomy tube (g-tube) placement (3), and premature birth is strongly associated with oropharyngeal and esophageal dysfunctions (4). Perinatal asphyxia patients with HIE have neuronal cell death and brain injuries, most commonly found in the basal ganglia and thalamus $(5,6)$. Perinatal injuries to certain parts of the brain such as the basal ganglia and thalamus have over 10-fold risk for g-tube, while solitary injury to the pons has 4-fold risk. Basal ganglia, thalamic, and pontine injury, combined, have a $91 \%$ probability of gastrostomy or long-term nasogastric tube (3). Advances in 
biomedical sciences have led to better diagnostic and therapeutic strategies among HIE infants admitted to neonatal ICUs; however, mechanisms of feeding impairment in those patients remain undefined (6).

Neurologic injuries have often been associated with aspiration, gastroesophageal reflux disease (GERD) and dysphagia in infants with feeding impairment. Abnormal oropharyngo-esophageal motility and brain-foregut interaction has been implicated as an underlying mechanism of these conditions $(7,8)$. We have previously shown modifications of adaptive responses following mechano-stimulation in infants with HIE (9). We have also shown that pharyngeal provocation results in altered proximal and distal esophageal peristalsis in HIE infants (10). These studies together support the assumptions that infants with HIE had prolonged adaptive responses to pharyngeal and esophageal provocation, aberrant upper esophageal sphincter (UES) function and decreased lower esophageal sphincter (LES) tone.

Healthy infants demonstrate maturational changes in pharyngo-esophageal protective reflexes over an interval of 2 to 4 weeks (10); however, development of such reflexes among infants with HIE remains unclear. Therefore, we tested the hypothesis that pharyngeal provocation-induced adaptive swallowing and UES contractile reflexes are distinct across maturation among infants with HIE, compared to control infants. Our aim was to evaluate the neurosensory and neuromotor aspects of pharyngeal provocation-induced adaptive reflexes in HIE infants across maturation. The response latency, response magnitude, and the frequency of recruitment of these reflexes among HIE infants were evaluated longitudinally at $41.4 \pm 0.6$ (HIE Time-1) and 46.5 \pm 0.6 weeks (HIE Time-2) post menstrual age (PMA).

\section{Materials and Methods}

\section{Subjects}

This study included fourteen infants diagnosed with HIE [7 males, $3.3 \pm 0.1 \mathrm{~kg} ; 39.8 \pm 0.4$ weeks gestation age (GA)] and seven control infants ( 3 males, $2.2 \pm 0.3 \mathrm{~kg} ; 34.6 \pm 1.4$ weeks GA). Infants were included in the HIE group if they were > 37 weeks GA, experienced acute perinatal asphyxia as defined by the presence of an acute perinatal event (placental abruption, cord prolapse, abnormal fetal heart rate), and presented with signs of encephalopathy per Sarnat staging (11). All infants with HIE underwent whole body therapeutic hypothermia (12). The control group included those who were born $>31.0$ weeks GA and had safe oral feeding skills at time of study and absence of HIE. Exclusion criteria for both groups included infants with > Grade $2 \mathrm{IVH}$ and/or periventricular leukomalacia, chromosomal anomalies, or gastrointestinal surgeries. The study procedures were approved by the Institutional Review Board at Nationwide Children's Hospital and the Health Insurance Portability and Accountability Acts (HIPAA). Informed consent from parents was obtained prior to study.

\section{Equipment and Study Protocol}

Solid state high resolution manometry (HRM) [Stationary Solar Gastro, Medical Measurement Systems (MMS) USA, Dover, NH] concurrent with provocative pharyngeal 
manometric methods were adapted (10,13-15). To perform the procedure, a 6-French solid state HRM catheter with 25 uni-directional pressure sensors spaced $1 \mathrm{~cm}$ apart (Unitip High Resolution Catheter, Unisensor USA, Portsmouth, $\mathrm{NH}$ ) was placed through the nare by a physician. The infant was then given time to adapt to catheter placement, and resting UES and esophagogastric junction (EGJ) high pressure zones were identified, and phases of spontaneous swallowing recognized. After determining the nares-UES upper border length, a specially designed 5.25 French silastic infusion catheter with a pharyngeal infusion port (Dentsleeve International, Mui Scientific, Ontario Canada) was positioned via the opposing nare, such that the pharyngeal infusion port was at $1 \mathrm{~cm}$ above the upper border of the UES. This catheter was then connected to a pressure transducer (TNF-R disposable, MMS USA, Dover $\mathrm{NH}$ ) to record the timing and duration of signal when infusion was given. Once again the infant was given time to adapt to catheter placement. Sequential graded pharyngeal infusions $(0.1,0.3$, and $0.5 \mathrm{ml}$, in triplicate) of saline or sterile water were abruptly administered by the physician. Dual band respiratory inductance plethysmography, nasal airflow thermistor, EKG, and pulse-oximetry were used to ensure patient safety.

\section{Data Analysis}

A total of 35 high resolution pharyngo-esophageal manometry studies ( 7 in controls, 14 in HIE Time-1 and 14 in HIE Time-2) were recorded. Responses to a total of 59 pharyngeal infusions in control infants and 241 infusions in HIE infants (119 for HIE Time-1 and 122 for HIE Time-2) were analyzed using MMS software (v. 8.23, MMS USA, Dover, NH). Utilizing both conventional HRM and esophageal pressure topography plots, manometric characteristics were analyzed using the following definitions (Figure 1): 1) UES resting tone $(\mathrm{mmHg})$ is defined as the average UES pressure during aerodigestive quiescence prior to pharyngeal provocation; 2) Primary response type (PRS, PUCR) is defined as any pharyngoesophageal activity occurring within 10 seconds of stimulus onset. PRS is defined as pharyngeal activity (or contractile waveforms in the pharyngeal region) associated with UES relaxation and deglutition apnea $(10,13,16)$. PUCR is defined as UES contractile activity or an increases of $\geq 4 \mathrm{mmHg}$ above UES resting tone $(10,13,16)$. If PRS was present, the number of pharyngeal peaks were counted, as multiple PRS responses can be expected with higher stimulus volume $(10,13,16) ; 3)$ Primary response latency (seconds) is defined as the duration between infusion onset to primary response onset (10); 4) Contractile front velocity (CFV) is defined as the tangential slope of the esophageal contraction along the $30 \mathrm{mmHg}$ isobaric contour (17); 5) Distal contractile integral (DCI; $\mathrm{mmHg}^{*} \mathrm{~cm}^{*} \mathrm{~s}$ ) is defined as amplitude*length*duration of the smooth muscle segment, calculated automatically based on marker placements at the transition zone to EGJ upper border. This is a measure of smooth muscle contractile vigor $(17-20)$; 6) Contractile vigor $\left(\mathrm{CV}, \mathrm{mmHg}^{*} \mathrm{~s}^{*} \mathrm{~cm}\right)$ is classified as failed (DCI is $<100 \mathrm{mmHg} * \mathrm{~s} * \mathrm{~cm}$ ), weak $(100-450 \mathrm{mmHg} * \mathrm{~s} * \mathrm{~cm}$ to 450 $\mathrm{mmHg} * \mathrm{~s} * \mathrm{~cm}$, or normal $(450 \mathrm{mmHg} * \mathrm{~s} * \mathrm{~cm}$ to $8000 \mathrm{mmHg} * \mathrm{~s} * \mathrm{~cm})(17,20) ; 7)$ Distal latency (DL; seconds) is defined as the duration between UES relaxation onset and the contractile deceleration point $(17,18)$; 8) Polymorphic waveform (PMW) is determined by the presence of 2 or more peaks in any single esophageal body channel (9);9) Terminal swallow as the final swallow that restores aerodigestive quiescence (21); 10) Terminal swallow latency (seconds) is defined as the duration from infusion onset to the onset of the terminal swallow; 11) Total response duration (seconds) is defined as the period from the 
onset of the infusion to the restoration of aerodigestive quiescence (22); 12) Threshold volume $(\mathrm{ml})$ is defined as the minimum volume of infusion eliciting either a PUCR or PRS response.

\section{Statistical Analysis}

Demographic characteristics were compared between HIE Time-1 and controls using unpaired $t$-tests, Wilcoxon rank sum and chi-squared tests. For comparison between HIE Time-1 and HIE Time-2, paired $t$-tests were used for continuous outcomes, and McNemar's test was used for categorical outcomes. Linear mixed models and generalized estimation of equations were used to compare manometric outcomes between HIE Time-1, HIE Time-2 and controls. Adjustment for multiple comparisons was made using the Bonferroni method. The two way mixed effects model was used to test the inter-rater reliability between investigators. The intraclass correlation coefficient (ICC) was calculated to be $99.5 \%$ (98.1 99.9; $p<0.001$ ), which indicate a high level of agreement between raters (MS and TRS). Results are presented as Mean \pm SEM, odds ratio (OR; 95\% CI) or percentage unless otherwise indicated. A $p$-value $<0.05$ was considered significant. Statistical analyses were performed using SAS software (version 9.3; SAS Institute Inc., Cary, NC, USA). ICC was calculated using SPSS (version 21; IBM Corp, Armonk, NY).

\section{Results}

Demographic characteristics for control and HIE infants at evaluation are described in table 1. Apgar scores for controls vs. HIE at 1 and 5 minutes, respectively, were 7 (6-8) vs. 2 (12) $(p=0.02)$ and $8(7-9)$ vs. $3(2-4)(p=0.01)$.

Of the 14 infants with HIE, two were classified as mild (Sarnat I), 11 moderate (Sarnat II), and one severe (Sarnat III). 28.6\% of HIE infants presented with clinical seizures. MRI data shows HIE-related abnormalities in $71.4 \%$ of HIE infants. At discharge, exclusive oral feeding was achieved by all 7 (100\%) controls and 13 (92.9\%) HIE infants.

\section{Effects of HIE on characteristics and distribution of the pharyngeal stimulus-induced reflexes}

Pharyngo-esophageal manometric data outcomes are presented in table 2 and figure 2, comparing HIE across maturation and vs controls. Briefly, threshold volume, response type and response latency were not significant across maturation or vs controls $(\mathrm{p}>0.05)$. However, with the presence of HIE, 1) UES resting pressure is increased; 2) number of pharyngeal peaks is decreased; 3) terminal swallow latency is decreased; 4) smooth muscle contractile vigor is abnormally weak or failed; and 5) distal latency is increased.

\section{Provocative Stimulus Volume-Response Relationships}

Using a stimulus-volume escalation protocol, we tested the effect on sensory-motor responses, and the odds of having a response and summarized the data in table 2. Increased frequency-recruitment of PRS responses at $0.3 \mathrm{ml}$ and $0.5 \mathrm{ml}$ (vs. $0.1 \mathrm{ml}$ ) for both controls and HIE Time- 2 infants were noted $(p<0.01)$. An increase in the number of pharyngeal peaks was evident with increasing volumes at $0.3 \mathrm{ml}$ among controls, HIE Time-1, and HIE 
Time- 2 , respectively $(6.0 \pm 1.1,3 \pm 0.5,2.9 \pm 0.3)$ vs. $0.1 \mathrm{ml}(1.4 \pm 1.2,1.7 \pm 0.5,1.4 \pm 0.4 ; p<0.05$ for comparison of $0.1 \mathrm{ml}$ vs. $0.3 \mathrm{ml}$ for all groups.).

\section{Discussion}

Normal maturation of aerodigestive functions among neonates is necessary for survival amidst dynamically-changing anatomy and rapidly evolving oro-pharyngo-esophageal protective mechanisms. In general, pharyngo-esophageal protective reflexes advance within a 2-4 week time window (10). However, during perinatal asphyxia, brain injury can be a critical barrier to neuronal and neuromuscular maturation, and this may contribute to delays with functional swallowing independence. The development of swallowing reflexes among HIE infants is not known. As maturation modifies the development of appropriate oropharyngo-esophageal mechanisms, we hypothesized that longitudinal maturation in infants with HIE will be different as these infants frequently have swallowing difficulties. Therefore, we investigated the longitudinal maturational trends of pharyngeal provocationinduced adaptive swallowing and UES reflexes in infants with HIE while comparing with oral-fed controls. This is the first study using provocative pharyngeal stimuli concurrent with high resolution manometry methods in infants.

Our data indicate that pharyngeal stimulus-induced reflexes are impaired in infants with HIE and are partially modified across five weeks of maturation. Despite full-term birth, perinatal asphyxia-induced HIE stuns the developmental progression of normal swallowing maturation, and accelerated strategies to optimize these and related reflexes are needed to ensure aerodigestive safety and oral feeding. Although the effect of maturation is the main research question in this study, it is important to note that for ethical reason we did not have a second longitudinal control group to match the longitudinal effect in HIE Time-2 patients.

Significantly, infants with HIE Time-1 (41.4 \pm 0.6 PMA) exhibited: 1) increased UES resting tone; 2) prolonged distal latency; and 3) decreased CFV. Prolonged distal latency and decreased velocity may be due to neuronal inflammation or neuronal apoptosis, which may have led to poor conductivity of afferent and/or efferent communicating neurons (23). Prolonged latency to primary response is seen consistently during maturation (HIE Time-2) in this study, suggesting lack of recovery of receptors or sensory pathways due to the HIE. Whereas when we analyzed the types and frequency of primary response to pharyngeal provocation, escalating stimulus volume-reflex responses were not statistically different between HIE Time-1 and HIE Time-2 (46.5 \pm 0.6 PMA). However, we found that the odds of having a PRS are 3.7 times with $0.3 \mathrm{ml}$ vs. odds of PRS with $0.1 \mathrm{ml}$ (Table 2). This phenomenon is not noted in HIE Time-1 group and could be attributed to impaired mechanosensitivity or osmo-sensitivity to escalating stimulus volumes. This finding also supports that greater stimulus magnitude (despite maturation) is needed to evoke swallowing reflexes at time-2, and may be related to partial recovery of neuromuscular functions. These alterations in stimulus-induced responses might be possible mechanisms for worsening feeding outcomes after NICU discharge, wherein $8 \%$ of infants who were successful feeders develop feeding problems as early as six months from discharge (6). Normalization of UES basal tone and CFV in HIE infants at $46.5 \pm 0.6$ PMA may be due to a decrease in inflammation and or down regulation of heightened cholinergic input to the skeletal and 
smooth muscles (24-26). Skeletal muscle hypertonicity along with smooth muscle hypotonicity was noted along with delays in distal latency. However, the number of pharyngeal waveforms was less and terminal swallow latency was shorter, thus suggesting that recovery of primary peristalsis mechanisms can occur over a period of $\sim 5$ weeks.

Although we have previously shown that infants with HIE have increased recruitment of PUCR as a primary response to pharyngeal provocation (15), the factors affecting maturation of this reflex in HIE requires further study. Primary responses to pharyngeal provocation involve both sensory and motor pathways. It is known that sensory receptors and pathways grow more rapidly than motor pathways (27). It is also known that corticospinal tracts become condensed at the level of the brainstem, (27) and could be influenced by diffuse cortical injury (11). Long term follow up studies are necessary to determine how enteric nervous system and autonomic nervous system adapt after HIE injury.

In our previous study (28), we have investigated pharyngeal stimulation-induced reflexes across healthy maturation and have found significant increase in PRS and liquid volumedependent increase in PRS recruitment frequency. Although adaptive aerodigestive protective responses seen in healthy maturation over a period of five weeks were noted previously by us (28), such observations are not evident in HIE patient upon maturation in the current study. Differences in excitatory and inhibitory modulation may be contributory in HIE during maturation $(15,28)$.

In conclusion, HIE infants showed increased UES basal tone, prolonged distal latency, and decreased CFV at HIE Time-1 when compared to controls. Moreover, with maturation (HIE Time-2), UES resting tone and distal latency were decreased, and increasing abnormal smooth muscle contractile vigor was observed, compared to HIE Time-1. Of note, these responses, except for abnormal contractile vigor, at HIE Time-2 were not significantly different from controls. Increased frequency-recruitment of PRS responses at $0.3 \mathrm{ml}$ and 0.5 $\mathrm{ml}$ (vs. $0.1 \mathrm{ml}$ ) for both controls and infants at HIE Time-2 were significant. On the other hand, a 5 week maturation period in infants with HIE seem to have no effect in pharyngeal recruitment, frequency of polymorphic waveforms, total latency, and the presence of terminal swallow. Perhaps, the five week maturational period is not adequate to determine whether all these variables will improve or worsen. This is in turn related to the complex interplay between the cortical, sub-cortical, and cerebellar connectivity necessary for suck, swallow, and peristalsis. Precise understanding of the mechanisms requires functional neuroimaging such as functional near infrared spectroscopy (29-31). Because dysphagia can be a surrogate clinical marker for aerodigestive dysfunctions and neurodevelopmental delays (32), developing neurosensory and neuromotor markers to identify these infants early could aid in mechanistic diagnosis as well as in optimizing therapies to improve feeding outcomes. We speculate that in doing so, short-term and long-term feeding milestones will advance when pathophysiology-based interventions are instituted. Adequately powered studies with longer interval of maturational period will give more insight on the timeline of how HIE or of its disease-severity may modify the progression or recovery of impaired swallowing. Accelerated strategies to optimize these and related reflexes are needed to achieve earlier and better functional aerodigestive outcomes. 


\title{
Acknowledgments
}

Supported in part by NIH grant R01 DK 068158 (Jadcherla). We are grateful to Rebecca Moore, BSN, RN for providing nursing observations and support.

Funding: Supported in part by grant funding from NIH-2R01DK068158 (Jadcherla).

\section{Abbreviations}

\author{
HIE Hypoxic Ischemic Encephalopathy \\ HIE Time-1 HIE studied at $41.4 \pm 0.6$ weeks PMA \\ HIE Time-2 HIE studied at $46.5 \pm 0.6$ weeks PMA \\ PMA Post Menstrual Age \\ PRS Pharyngeal Reflexive Swallow \\ PUCR Pharyngo-Upper Esophageal Sphincter Contractile Reflex \\ UES Upper Esophageal Sphincter
}

\section{References}

1. Hamilton BE, Martin JA, Ventura SJ. Births: preliminary data for 2012. National vital statistics reports: from the Centers for Disease Control and Prevention, National Center for Health Statistics, National Vital Statistics System. 2013; 62:1-20.

2. Martin JA, Hamilton BE, Ventura SJ, Osterman MJ, Mathews TJ. Births: final data for 2011. National vital statistics reports: from the Centers for Disease Control and Prevention, National Center for Health Statistics, National Vital Statistics System. 2013; 62:1-69. 72.

3. Martinez-Biarge M, Diez-Sebastian J, Wusthoff CJ, et al. Feeding and communication impairments in infants with central grey matter lesions following perinatal hypoxic-ischaemic injury. European journal of paediatric neurology: EJPN: official journal of the European Paediatric Neurology Society. 2012; 16:688-696. [PubMed: 22658307]

4. Rommel N, De Meyer AM, Feenstra L, Veereman-Wauters G. The complexity of feeding problems in 700 infants and young children presenting to a tertiary care institution. J Pediatr Gastroenterol Nutr. 2003; 37:75-84. [PubMed: 12827010]

5. Malkar MB, Jadcherla S. Neuromotor mechanisms of pharyngoesophageal motility in dysphagic infants with congenital heart disease. Pediatr Res. 2014; 76:190-196. [PubMed: 24819378]

6. Van Houten JP, Benaron DA, Spilman S, Stevenson DK. Imaging brain injury using time-resolved near infrared light scanning. Pediatr Res. 1996; 39:470-476. [PubMed: 8929868]

7. Pensabene L, Miele E, DelGiudice E, Strisciuglio C, Staiano A. Mechanisms of gastroesophageal reflux in children with sequelae of birth asphyxia. Brain \& Development. 2008; 30:563-571. [PubMed: 18328656]

8. Andrew MJ, Parr JR, Sullivan PB. Feeding difficulties in children with cerebral palsy. Arch Dis Child Educ Pract Ed. 2012

9. Hill CD, Jadcherla SR. Esophageal mechanosensitive mechanisms are impaired in neonates with hypoxic-ischemic encephalopathy. J Pediatr. 2013; 162:976-982. [PubMed: 23260103]

10. Jadcherla SR, Shubert TR, Gulati IK, Jensen PS, Wei L, Shaker R. Upper and Lower Esophageal Sphincter Kinetics are Modified During Maturation: Effect of Pharyngeal Stimulus in Premature Infants. Pediatr Res. 2014

11. Sarnat H, Sarnat M. Neonatal encephalopathy following fetal distress. A clinical and electroencephalographic study. Arch Neurol. 1976; 33:696-705. [PubMed: 987769] 
12. Shankaran S, Laptook AR, Ehrenkranz RA, et al. Whole-body hypothermia for neonates with hypoxic-ischemic encephalopathy. N Engl J Med. 2005; 353:1574-1584. [PubMed: 16221780]

13. Jadcherla SR, Gupta A, Stoner E, Fernandez S, Shaker R. Pharyngeal swallowing: defining pharyngeal and upper esophageal sphincter relationships in human neonates. J Pediatr. 2007; 151:597-603. [PubMed: 18035137]

14. Jadcherla SR, Gupta A, Wang M, Coley BD, Fernandez S, Shaker R. Definition and implications of novel pharyngo-glottal reflex in human infants using concurrent manometry ultrasonography. Am J Gastroenterol. 2009; 104:2572-2582. [PubMed: 19603008]

15. Gulati IK, Shubert TR, Sitaram S, Wei L, Jadcherla SR. Effects of birth asphyxia on the modulation of pharyngeal provocation-induced adaptive reflexes. American journal of physiology Gastrointestinal and liver physiology. 2015; 309:G662-669. [PubMed: 26272260]

16. Jadcherla SR, Hasenstab KA, Sitaram S, Clouse BJ, Slaughter JL, Shaker R. Effect of Nasal NonInvasive Respiratory Support Methods on Pharyngeal Provocation Induced Aero-digestive Reflexes in Infants. Am J Physiol Gastrointest Liver Physiol. 2016 ajpgi 0030702015.

17. Jobsis FF. Noninvasive, infrared monitoring of cerebral and myocardial oxygen sufficiency and circulatory parameters. Science. 1977; 198:1264-1267. [PubMed: 929199]

18. Johnson MD, Piech K, Emandian S. Radiation-associated meningioma in the elderly: development of meningioma with olfactory neuroblastoma recurrence 10 years after irradiation. Annals of clinical and laboratory science. 2013; 43:460-463. [PubMed: 24247807]

19. Staiano A, Boccia G, Miele E, Clouse RE. Segmental characteristics of oesophageal peristalsis in paediatric patients. Neurogastroenterol Motil. 2008; 20:19-26. [PubMed: 18031473]

20. Shubert TR, Sitaram S, Jadcherla SR. Effects of pacifier and taste on swallowing, esophageal motility, transit, and respiratory rhythm in human neonates. Neurogastroenterol Motil. 2016; 28:532-542. [PubMed: 26727930]

21. Jadcherla SR, Hasenstab KA, Sitaram S, Clouse BJ, Slaughter JL, Shaker R. Effect of nasal noninvasive respiratory support methods on pharyngeal provocation-induced aerodigestive reflexes in infants. Am J Physiol Gastrointest Liver Physiol. 2016; 310:G1006-1014. [PubMed: 27012774]

22. Hasenstab KA, Jadcherla SR. Respiratory events in infants presenting with apparent life threatening events: is there an explanation from esophageal motility? J Pediatr. 2014; 165:250 255. e251. [PubMed: 24681180]

23. Volpe JJ. Brain injury in premature infants: a complex amalgam of destructive and developmental disturbances. Lancet Neurol. 2009; 8:110-124. [PubMed: 19081519]

24. Jadcherla SR. Inflammation inhibits muscarinic signaling in in vivo canine colonic circular smooth muscle cells. Pediatr Res. 2002; 52:756-762. [PubMed: 12409525]

25. Kahrilas PJ, Boeckxstaens G. The spectrum of achalasia: lessons from studies of pathophysiology and high-resolution manometry. Gastroenterology. 2013; 145:954-965. [PubMed: 23973923]

26. Anju TR, Naijil G, Shilpa J, Roshni T, Paulose CS. Neonatal hypoxic insult-mediated cholinergic disturbances in the brain stem: effect of glucose, oxygen and epinephrine resuscitation. Neurological sciences: official journal of the Italian Neurological Society and of the Italian Society of Clinical Neurophysiology. 2013; 34:287-296.

27. Volpe, JJ. Neurology of the Newborn. 5. Philadelphia: Philadelphia: Elsevier; 2008.

28. Jadcherla SR, Shubert TR, Gulati IK, Jensen PS, Wei L, Shaker R. Upper and lower esophageal sphincter kinetics are modified during maturation: effect of pharyngeal stimulus in premature infants. Pediatr Res. 2015; 77:99-106. [PubMed: 25279989]

29. Jadcherla SR, Pakiraih JF, Hasenstab KA, et al. Esophageal reflexes modulate frontoparietal response in neonates: Novel application of concurrent NIRS and provocative esophageal manometry. Am J Physiol Gastrointest Liver Physiol. 2014; 307:G41-49. [PubMed: 24789204]

30. Kashou NH, Giacherio BM, Nahhas RW, Jadcherla SR. Hand-grasping and finger tapping induced similar functional near-infrared spectroscopy cortical responses. Neurophotonics. 2016; 3:025006. [PubMed: 27335888]

31. Kashou NHD, IH K, Nahhas R, Jadcherla SR. Somatic Stimulation Causes Fronto-Parietal Cortical Changes in Neonates: an fNIRS Study. Neurophotonics. In Press 
32. Slattery J, Morgan A, Douglas J. Early sucking and swallowing problems as predictors of neurodevelopmental outcome in children with neonatal brain injury: a systematic review. Dev Med Child Neurol. 2012; 54:796-806. [PubMed: 22607330] 


\section{Key Points}

- Maturation affects pharyngo-esophageal protective reflexes in healthy infants. The development of such reflexes among survivors of hypoxic ischemic encephalopathy infants (HIE) is unclear. We evaluated these reflexes (longitudinally) in HIE infants following pharyngeal stimulation.

- $\quad$ High resolution manometry analysis showed that pharyngo-esophageal protective reflexes are impaired in HIE infants which may be responsible for inadequate clearance of secretions, ascending refluxate, and oropharyngeal bolus.

- Strategies to optimize these reflexes are needed to ensure aerodigestive safety during oral feeding. 


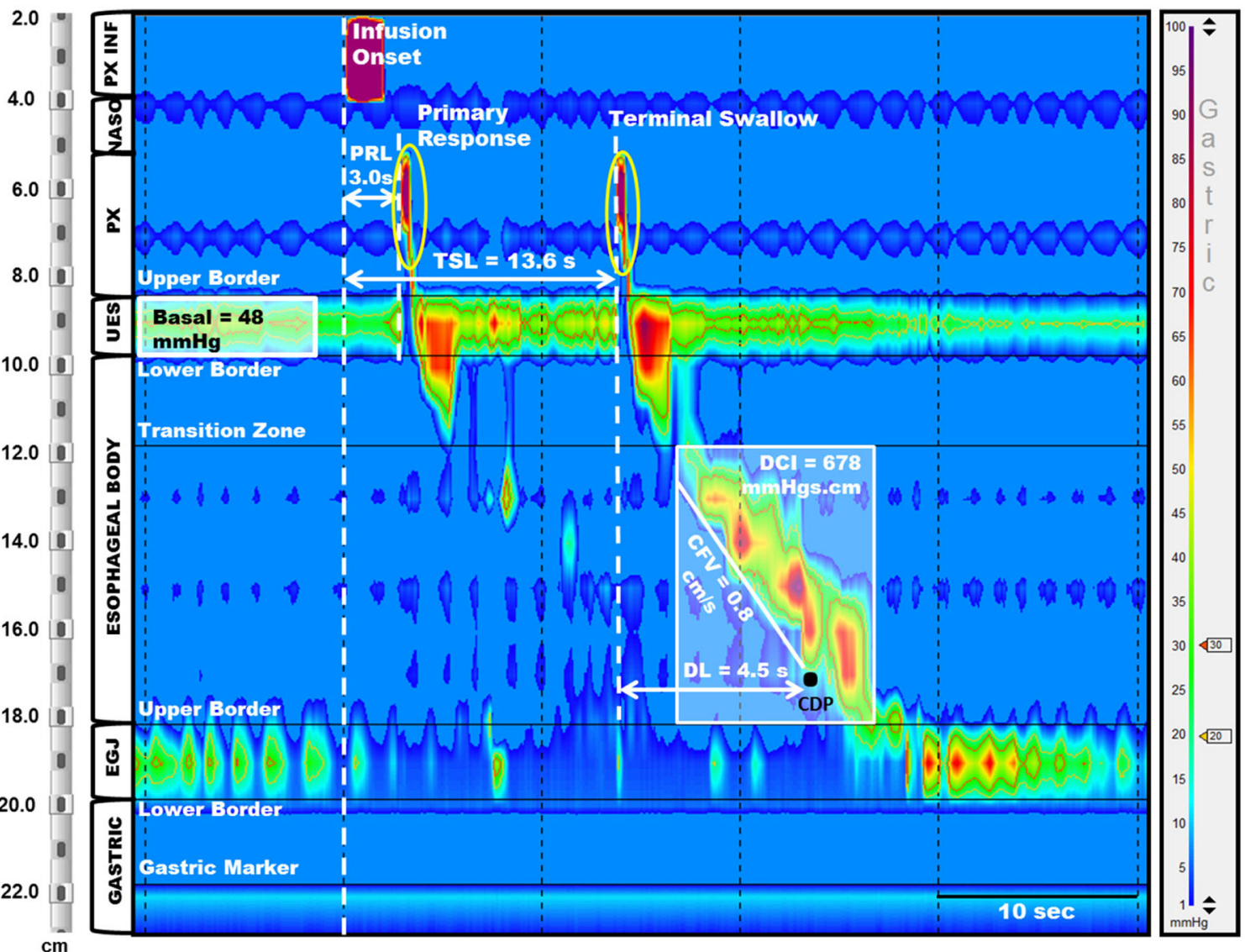

Figure 1. Esophageal Pressure Topography Definitions

Px Inf- Pharyngeal Infusion, Naso- Nasal cavity, Px- Pharynx, UES- Upper Esophageal

Sphincter, EGJ- Esophago Gastric Junction. This is a representative example of the analytical definitions utilized using esophageal pressure topography: A) Basal UES pressure, $\mathrm{mmHg}$, as average UES pressure prior to pharyngeal infusion, B) Primary Response Latency (PRL, sec) as duration of infusion onset to primary response onset, C) Terminal Swallow as the final swallow that restores aerodigestive normalcy, D) Terminal Swallow Latency (TSL, sec) as duration of infusion onset to terminal swallow onset E) Distal Latency (DL, sec) as duration of terminal swallow onset to contractile deceleration point (CDP), F) Contractile Front Velocity (CFV, $\mathrm{cm} / \mathrm{s}$ ) as the tangential slope of the $30 \mathrm{mmHg}$ isobar in the smooth muscle segment located by the upper border of the transition zone to the lower border of the EGJ, G) the Distal Contractile Integral calculated automatically by MMS software. The number of pharyngeal peaks or peristaltic patterns can be counted are highlighted in yellow circles. 
A

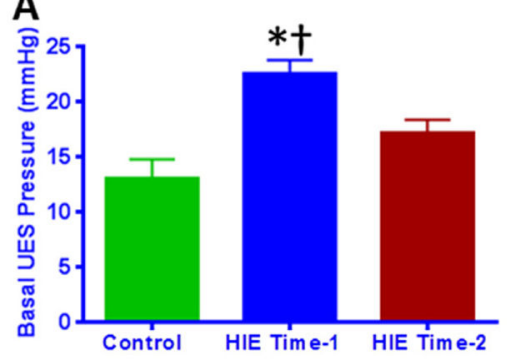

C

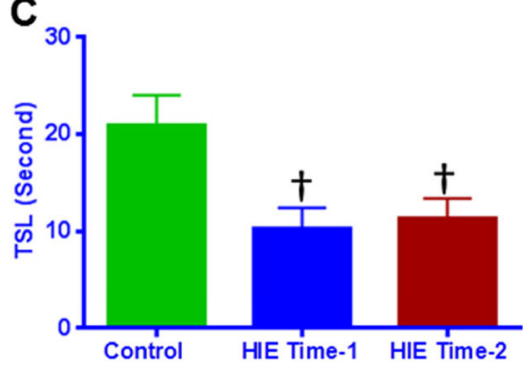

E

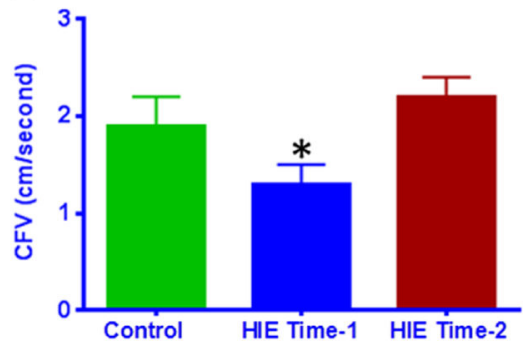

B
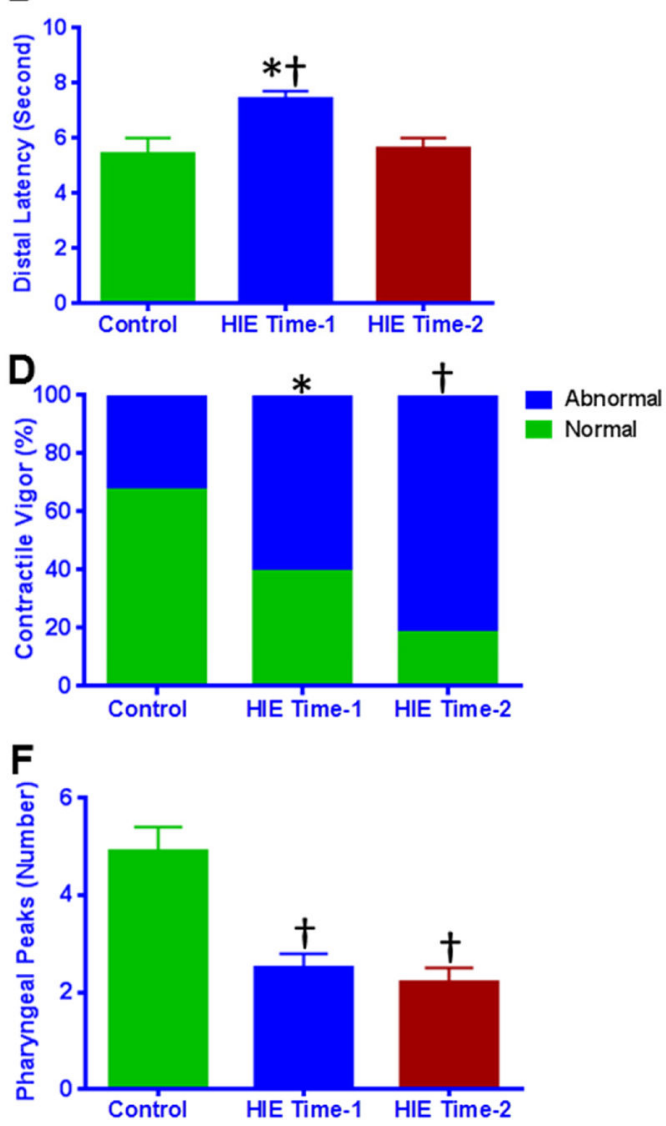

Figure 2. Pharyngo-Esophageal Characteristics in Hypoxic Ischemic Encephalopathy Across Maturation

A) UES basal pressure is increased at HIE time-1. B) Esophageal distal is increased at HIE time-1. C) Terminal swallow latency is decreased in HIE. D) Smooth muscle contractile vigor is abnormal (weak or failed) in HIE. E) Contractile front velocity is increased with maturation in HIE. F) The number of pharyngeal peaks in response to pharyngeal stimulation is decreased in HIE.

HIE: Hypoxic Ischemic Encephalopathy, HIE Time-1: Studied at 41.4 \pm 0.6 weeks PMA, HIE Time-2: Studied at $46.5 \pm 0.6$ weeks post menstrual age. Data presented as Mean \pm SEM or $\%$.

$* \mathrm{p}<0.05$ vs HIE Time- $2 . \dagger \mathrm{p}<0.05$ vs controls. 


\section{Table 1}

Demographic and Growth Characteristics at Time of Evaluation

\begin{tabular}{llll}
\hline Characteristics & Controls & HIE Time-1 & HIE Time-2 \\
\hline Frontal-Occipital Circumference, cm & $35.6 \pm 0.4$ & $34.2 \pm 0.4^{\dagger}$ & $36.8 \pm 0.3^{*}$ \\
Weight, kg & $3.9 \pm 0.3$ & $3.5 \pm 0.1^{\dagger}$ & $4.5 \pm 0.1$ \\
Post Menstrual Age, weeks & $43.5 \pm 1.3$ & $41.4 \pm 0.6^{\dagger}$ & $46.5 \pm 0.6^{*}$ \\
Chronological Age, weeks & $7.0(7.0-11.0)$ & $1.2(1.0-2.0)^{*}{ }^{\dagger}$ & $6.6(5.7-7.8)^{*}$ \\
Length, cm & $52.6 \pm 1.1$ & $51.5 \pm 0.7$ & $54.5 \pm 0.7$ \\
\hline
\end{tabular}

HIE: Hypoxic Ischemic Encephalopathy, HIE Time-1: Studied at 41.4 \pm 0.6 weeks PMA, HIE Time-2: Studied at $46.5 \pm 0.6$ weeks post menstrual age. Data presented as Mean \pm SEM or median (interquartile range). $t$-test was used to compare demographic characteristics.

*

: denotes significance from controls at $p<0.05$.

${ }^{\dagger}$ : denotes significance from HIE Time-2 at $p<0.01$. 


\section{Table 2}

Pharyngo-Esophageal Characteristics in Hypoxic Ischemic Encephalopathy Across Maturation

\begin{tabular}{llll}
\hline Characteristics & Controls (N=59 Stimuli) & HIE Time-1 (N=119 Stimuli) & HIE Time-2 (N=122 Stimuli) \\
\hline Response (PRS: PUCR: None), \% & $56: 18: 26$ & $50: 17: 33$ & $53: 13: 34$ \\
PRL; Seconds & $3.9 \pm 0.5$ & $4.3 \pm 0.4$ & $4.9 \pm 0.4$ \\
Terminal Swallow (Yes); \% & 72 & 85 & 88 \\
DCI, mmHg.cm.s & $536.9 \pm 88.5$ & $406.8 \pm 60.5$ & $294.3 \pm 60$ \\
Abnormal CV (Failed: Weak); \% & $63: 37$ & $34: 66$ & $31: 69$ \\
DCIa, mmHg.s & $65.0 \pm 10.4$ & $50.6 \pm 7^{*}$ & $32.8 \pm 7^{\dagger}$ \\
PMW (Yes), \% & 27.30 & 42.40 & 28.40 \\
Threshold volume, $\mathrm{mL}$ & $0.2 \pm 0.02$ & $0.2 \pm 0.02$ & $0.2 \pm 0.02$ \\
\hline
\end{tabular}

HIE: Hypoxic Ischemic Encephalopathy, HIE Time-1: Studied at 41.4 \pm 0.6 weeks PMA, HIE Time-2 Studied at $46.5 \pm 0.6$ weeks post menstrual age.

PRS: Pharyngeal Reflexive Swallow; PUCR: Pharyngo-Upper Esophageal Sphincter Contractile Reflexes; PRL: Primary response latency; DCI: Distal contractile integral; CV: Contractile vigor; PMW: Polymorphic waveform. Data presented as Mean \pm SEM or \%.

*

* denotes significance from HIE Time-1 at $\mathrm{p}<0.05$.

${ }^{\dagger}$ : denotes significance from controls at $\mathrm{p}<0.05$ 


\section{Table 2}

\section{Stimulus Response Relationships Across Maturation}

\begin{tabular}{lllll}
\hline & & \multicolumn{3}{c}{ Odds Ratio (95\% CI) } \\
\cline { 3 - 5 } Volume (ml) & Response & Control & HIE: Time-1 & HIE: Time-2 \\
\hline 0.1 vs. 0.3 & -Pharyngo-Upper Esophageal Sphincter Contractile Reflexes & $0.6(0.2-1.7)$ & $0.8(0.3-1.9)$ & $1.1(0.4-3.3)$ \\
& -Pharyngeal Reflexive Swallow & $7.8(2.6-23.5)^{*}$ & $2.1(1-4.6)$ & $3.7(1.8-7.5)^{*}$ \\
& -No Response & $0.1(0.02-0.6)^{\dagger}$ & $0.5(0.2-1.0)$ & $0.2(0.1-0.5)^{*}$ \\
\cline { 2 - 5 } 0.3 vs. 0.5 & -Pharyngo-Upper Esophageal Sphincter Contractile Reflexes & $1.1(0.2-5.6)$ & $0.8(0.3-2.1)$ & $0.9(0.2-3.4)$ \\
& -Pharyngeal Reflexive Swallow & $0.7(0.2-2.3)$ & $0.7(0.3-1.4)$ & $0.6(0.2-1.4)$ \\
& -No Response & $1.8(0.4-7.6)$ & $1.8(0.9-3.5)$ & $2.1(0.9-4.9)$ \\
\cline { 2 - 5 } 0.1 vs. 0.5 & -Pharyngo-Upper Esophageal Sphincter Contractile Reflexes & $0.7(0.3-1.5)$ & $0.6(0.2-2.4)$ & $1(0.2-3.9)$ \\
& -Pharyngeal Reflexive Swallow & $5.2(1.8-15.3)^{*}$ & $1.5(0.5-4.3)$ & $2.2(1-5)$ \\
& -No Response & $0.2(0.09-0.5)^{*}$ & $0.8(0.3-2.5)$ & $0.5(0.2-1.2)$ \\
\hline
\end{tabular}

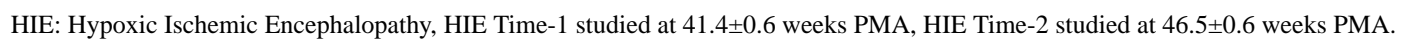

The association between independent variables (Volume) and dependent variables (Response) were measured using generalized estimating equation methods. Odds ratio > 1 denotes an increase in odds of having a response at higher volumes vs. lower volumes. For example, at Time- 2 , the odds of having a pharyngeal reflexive swallow response at $0.3 \mathrm{ml}$ is 3.7 times the odds of having the same response at $0.1 \mathrm{ml}$. Odds ratio $<1$ denotes a decrease in odds of having a response at higher volumes vs. lower volumes. For example, among controls, the odds of having a no response at 0.3 $\mathrm{ml}$ is 0.1 times the odds of having no response at $0.1 \mathrm{ml}$.

Data presented as Mean \pm SEM or median (interquartile range)

* denotes significance at $p<0.01$

$t^{\dagger}$ denotes significance at $p<0.05$. 